\title{
Correction to: Next-generation immunotherapy for pancreatic ductal adenocarcinoma: navigating pathways of immune resistance
}

\author{
Thatcher Heumann ${ }^{1} \cdot$ Nilofer Azad ${ }^{1}$
}

Published online: 19 October 2021

๑) Springer Science+Business Media, LLC, part of Springer Nature 2021

\section{Correction to: Cancer and Metastasis Reviews https://doi.org/10.1007/s10555-021-09981-3}

The original published version of this article contained a mistake in the figure captions. The figure captions captured the title of the figures, but the figure descriptions were not included. Below are the correct figure captions:

Fig. 1 Adaptive Immune Response inCancer.Immunotherapyrelies on the ability of host immune cells to recognize cancer as cells thatneed to be eliminated. For this to happen, a tumor cell needs to alertsurveilling antigen presenting cells (APCs), such as dendritic cells (DC), thatthey are abnormal by expressing antigens on their cell surface. When APCsrecognize this abnormal signal, they take up the antigen, traffic to lymphnodes to mature and, in turn, activate cytotoxic and helper T cells with T cellreceptors (TCR) specific to that tumor antigen. Once these T cells areactivated, they should further differentiate, expand, and migrate to the tumorbed and kill that tumor cell. Adapted from "Antigen Presentation in Cancer", byBioRender.com (2021). Retrieved from https://app.biorender.com/biorender-templates.

Fig. 2 Mechanisms of ImmuneResistance in Pancreatic Cancer.Pancreatic cancer(PDAC) has several protections against the generation and execution of ananti-tumor hostimmune response. A) PDAC's low antigen burden, sequestration ofMHC/HLA-I molecules, and local tolerogenic TME signaling all contribute to poorimmune surveillance and stunt normal dendritic cell maturation. Without

The original article can be found online at https://doi.org/10.1007/ s10555-021-09981-3.

Nilofer Azad

Nazad2@jhmi.edu

1 Department of Medical Oncology, Sidney Kimmel Comprehensive Cancer Center, Johns Hopkins University School of Medicine, Baltimore, MD, USA normalantigen processing and presentation, anti-tumor effectors are not activated. B)If activation and clonal expansion of effector T cells still does manage tooccur, trafficking to the tumor bed is complicated by disrupted chemokinesgradients, abnormal vasculature, and the desmoplastic tumor stroma that acts asboth a physical \& chemical barrier to entry. C) Anti-tumor immune cellsthat are able to penetrate the TME are quickly exhausted by local metabolicconditions combined with immunosuppressive molecular crosstalk (e.g.upregulated interleukin 1 beta [IL-1b ],transforming growth factor beta [TGFb],interleukin 10 [IL-10], and beta-catenin [b-catenin]) between tumor and tumor-coopted immune cell populations(cancer-associated fibroblasts [CAF]; tumor associated macrophages [TAM]; tumorassociated neutrophils [TAN]; tolerogenic dendritic cells [DC]; myeloid derivedsuppressor cells [MDSC]; type II $\mathrm{T}$ helper cells [Th2]; T regulatory cells[Treg]; B regulatory cells [Breg]). Adapted from "Challenges for CAR T-CellImmunotherapy in Solid Tumors" and "Tumor Extracellular Matrix ReducesTherapeutic Efficiency in Solid Tumors", by BioRender.com (2021). Retrievedfrom https://app.biorender. com/biorender-templates.

Fig. 3 A Framework for SuccessfulIO-based Treatment in PDAC.To block immuneescape, next generation IObased approaches for PDAC must be built on targetingimmune priming/activation pathways simultaneously with modulating local TMEimmunosuppression and blocking immune checkpoints blockade for effectivesustained antitumor immune trafficking and sustained cytotoxic response. Thiswill require several IO and targeted agents on top of conventional therapies.Standard chemotherapy with combinatorial cocktail of an anti-tumor vaccine,co-stimulatory agent (aCD40), TME crosstalk modulator (CXC4Ri or $\mathrm{TGFb}$ trap), and ICB (aPD-1) is one such potential combination. Key(selected terms): [cDC] conventional dendritic cell; [CSF-1Ri]colony-stimulating factor-1 receptor inhibitor; [CTL] cytotoxic T lymphocyte;[CTLA-4] cytotoxic T lymphocyte antigen 4; [DDRi] DNA damage repair 
inhibitor;[DNMTi] DNA methyltransferase inhibitor; [FAKi] focal adhesion kinaseinhibitor; [FLT3L] Fms-related receptor tyrosine kinase 3; [HDACi] histonedeacetylase inhibitor; [Hyals] hyalanurinases; [LAG3] lymphocyte activating 3 ;[M1] tumor associated macrophage, type I phenotype; [mAbs] monoclonal antibodies;[N1] Neutrophil, type 1 phenotype; [NK] - natural killer; [NK1] natural killercell, type 1 phenotype; [PARPi] Poly (ADP-ribose) polymerase inhibitor; [PD-L1]- programmed death-ligand 1; [RT] radiation therapy; [TGFb] transforming growth factor beta; [Th1] T helper cell, type 1phenotype; [VEGFi] Vascular endothelial growth factor inhibitor. Created withBioRender.com.

The original article has been corrected.

Publisher's note Springer Nature remains neutral with regard to jurisdictional claims in published maps and institutional affiliations. 